\title{
The influence of a 3-week body mass reduction program on the metabolic parameters and free amino acid profiles in adult Polish people with obesity
}

\author{
Małgorzata Moszak ${ }^{1, A-D}$, Agnieszka Klupczyńska ${ }^{2, A-D}$, Alina Kanikowska, ${ }^{1, A, B, D, E}$, Zenon Kokot ${ }^{2, A, C, E, F}$, \\ Agnieszka Zawada ${ }^{1, B, C}$, Małgorzata Grzymisławska ${ }^{3, B, C}$, Marian Grzymisławski ${ }^{1, A, C, E, F}$ \\ ${ }^{1}$ Department of Gastroenterology, Human Nutrition and Internal Diseases, Poznan University of Medical Sciences, Poland \\ 2 Department of Inorganic and Analytical Chemistry, Poznan University of Medical Sciences, Poland \\ ${ }^{3}$ Department and Chair of Anatomy, Poznan University of Medical Sciences, Poland \\ A - research concept and design; B - collection and/or assembly of data; $\mathrm{C}$ - data analysis and interpretation; \\ $D$ - writing the article; $E$ - critical revision of the article; $F$ - final approval of the article
}

Address for correspondence

Małgorzata Moszak

E-mail:mmoszak@ump.edu.pl

Funding sources

None declared

Conflict of interest

None declared

Received on January 9, 2017

Reviewed on January 31,2017

Accepted on April 27, 2017

\begin{abstract}
Background. Previous studies have shown differences in the amino acid (AA) composition in the plasma of people with obesity when compared to lean individuals, but the perturbations of AA concentrations in obesity and the dynamics of AA changes after weight loss are not fully understood.

Objectives. The objective of the study was to evaluate the effect of a short-term weight reduction program on the metabolic status and plasma AA levels in individuals with obesity.

Material and methods. A total of 24 adult Polish patients with a BMl between 34 and $49 \mathrm{~kg} / \mathrm{m}^{2}$ were enrolled in a 3-week controlled body mass reduction program based on everyday physical activity and a hypocaloric diet (25-30\% less than total daily energy requirements). At baseline and after the program, anthropometric measurements, biochemical parameters and free AA profiles were determined.

Results. After the weight loss program, significant changes in body mass and metabolic parameters (e.g., low-density lipoprotein, triglyceride, fasting glucose, and insulin levels) were observed. Positive changes in a homeostatic model assessment of insulin resistance (HOMA-IR) and quantitative insulin sensitivity check index (QUICKI) following the program were also found. The levels of 10 AAs (a-amino-n-butyric acid, alanine, citrulline, glutamine, glycine, hydroxyproline, isoleucine, proline, sarcosine, and threonine) had significantly increased following weight loss. Only aspartic acid was present at a significantly lower concentration after the program.

Conclusions. Using a 3-week controlled body mass reduction program based on physical activity and a hypocaloric diet, we were able to demonstrate significant changes in biochemical parameters and free AA profiles. To better understand these changes, future studies should involve a long-term program with more patients.
\end{abstract}

Key words: obesity, amino acids, metabolic profile, body mass reduction

DOI

10.17219/acem/70796

Copyright

Copyright by Author(s)

This is an article distributed under the terms of the

Creative Commons Attribution Non-Commercial License

(http://creativecommons.org/licenses/by-nc-nd/4.0/) 


\section{Introduction}

Obesity is a significant public health problem reaching the level of pandemic in the developed world. It not only predisposes individuals to serious chronic conditions, such as type 2 diabetes, cardiovascular diseases and certain cancers, but it is also a leading cause of premature death worldwide. The etiology of obesity is multifactorial and includes interactions between genetic and environmental factors. Obesity results from energy homeostasis disorders, caused by an excessive intake of calories in relation to energy expenditure, and other lifestyle-related factors, such as a sedentary job and inactivity. ${ }^{1,2}$

In recent years, there has been a growing interest in the study of the human metabolome in obesity. The term "metabolome" is defined as the complete set of all metabolites in the body, tissues or cells (e.g., amino acids, lipids, carbohydrates, or nucleotides). ${ }^{3}$ Free amino acids (AAs) constitute a particularly interesting group of metabolites. ${ }^{4,5}$

Previous studies showed significant differences in plasma AA composition in people with obesity compared to individuals with normal body mass. ${ }^{6-9}$ Newgard et al. observed that among a total of 16 AAs measured in the serum of adult African-American subjects, 8 amino acids (alanine, valine, leucine/isoleucine, phenylalanine, tyrosine, glutamate/glutamine, aspartate/asparagine, and arginine) were found in dramatically higher levels in obese participants (median body mass index - BMI: $36.6 \mathrm{~kg} / \mathrm{m}^{2}$ ) vs lean participants (median BMI: $23.2 \mathrm{~kg} / \mathrm{m}^{2}$ ). ${ }^{10}$ Only the glycine level was lower in patients with obesity.

Strong discrepancies between the branched-chain amino acid (BCAA) and AA levels in children with obesity (8-18 years old) compared to lean individuals were also described by McCormack et al. ${ }^{11}$ A report on Japanese subjects with obesity showed that plasma levels of alanine, glycine, glutamate, tryptophan, tyrosine, and BCAAs were associated with high visceral fat accumulation and could be dependent on genetic and environmental factors. ${ }^{12}$ Moreover, Yamakado et al. hypothesized that the amount of visceral fat changes AA profile, and that the multivariate logistic regression model of free AAs in plasma can discern non-apparent visceral obesity in adult Asian individuals. ${ }^{12}$ The associations between the BCAA levels and tyrosine with visceral adiposity - irrespective of ethnicity, lifestyle or environmental conditions - were also studied by Marti et al. ${ }^{13}$

Despite the great interest in and development of the state of knowledge about metabolic profiles in people with obesity, the perturbations in AA concentrations in the serum of adult patients suffering from obesity after weight loss are not fully understood. The previous studies in this area focused on describing the changes in metabolic profile after weight loss achieved by bariatric surgery or longterm body mass reduction programs based on lifestyle intervention. In addition, previous studies evaluated the AA changes in groups of people of different races or with different baseline BMI levels, and used other metabolomic assay techniques. Only a few studies investigated the effect of weight reduction on the level of amino acids in time points shorter than 1 year on a Caucasian population. An intriguing issue is the dynamics of changes in the metabolome under weight loss intervention.

This is the first study which demonstrates alterations in AA profiles and a number of biochemical parameters: fasting serum glucose, fasting serum insulin and a homeostatic model assessment of insulin resistance (HOMAIR), quantitative insulin sensitivity check index (QUICKI), C-reactive protein (CRP), total cholesterol (TC), high-density lipoprotein (HDL), low-density lipoprotein (LDL), triglyceride (TG), alanine aminotransferase (AlAT), and aspartate aminotranferase (AspAT) after a 3-week program based on a controlled, energy-restricted diet (25-30\% less than daily energy requirements) of a strictly specified macronutrient composition and physical activity. The panel of free AAs analyzed was the most comprehensive among all previously conducted studies and it covered concentrations of 42 metabolites.

\section{Material and methods}

\section{Participants and study design}

The study was conducted in the Department of Internal Diseases, Metabolism and Nutrition of the Heliodor Swiecicki University Hospital in Poznań, Poland, between September 2014 and June 2015. Twenty-four Polish patients (10 men and 14 women) with a BMI between 34 and $49 \mathrm{~kg} / \mathrm{m}^{2}$ (mean BMI: $40 \pm 4 \mathrm{~kg} / \mathrm{m}^{2}$ ) were enrolled. Mean age was $46 \pm 12$ years and ranged from 24 to 66 years. Diabetes mellitus type 1 or uncontrolled type 2 diabetes, a vegetarian or any other alternative diet, cancer, a history of eating disorders, or chronic diseases related to metabolism (e.g., chronic liver, kidney or pancreatic diseases, inborn metabolic diseases, autoimmune diseases, or inflammatory bowel diseases) were considered to be exclusion criteria. The occurrence of diseases listed in the exclusion criteria was checked through the use of specific diagnostic tests and medical histories. None of the patients smoked.

The study protocol was approved by the Bioethics Committee at Poznan University of Medical Sciences (No. 333/14), and was performed in accordance with the Declaration of Helsinki. All the patients signed statements of informed consent before they participated in the study.

The subjects were hospitalized for 3 weeks and received controlled daily aerobic physical training, under the supervision of a physical therapist, and a hypocaloric diet based on a 25-30\% reduction of caloric dietary intake compared to their total estimated energy requirement. The total daily energy requirement was calculated on actual body weight using the Harris-Benedict formula and the physical activity level index (PAL). To more accurately determine total 
energy expenditure and to assess the caloric value of the diet before the program, subjects completed a 3-day intake assessment prior to the study (the 24-hour dietary assessment covers 3 days). The subjects received the same type of diet prepared by dietetic food caterers based on the planned menu. The average value of total daily energy intake before the program was $2,935.6 \pm 490.2 \mathrm{kcal}$ $(2,764.0 \pm 406.1 \mathrm{kcal}$ for women; 3,340.5 \pm 284.5 for men), and the average value during the body mass reduction program was $2,016.0 \pm 281.7 \mathrm{kcal}(1,880.0 \pm 234.0 \mathrm{kcal}$ for women; 2,220.0 \pm 219.1 for men).

Each patient received a diet with an identical composition of macronutrients (especially proteins) derived from the same products. The diet consisted of $20 \%$ of calories from protein, $25-30 \%$ from fat, and 50-55\% from carbohydrates. The daily fiber intake from the diet was over $25 \mathrm{~g}$. Physical training included active and passive breathing exercises for $30 \mathrm{~min}$ a day, cardiovascular aerobic exercise (Nordic walking or cycling) twice a day for $60 \mathrm{~min}$ and resistance training for $30 \mathrm{~min}$ daily. Each patient who participated in the study was educated on proper nutrition and nutritional recommendations for obesity by a qualified nutritionist.

\section{Serum assays}

Serum glucose (3.3-5.8 mmol/L), TC (3.4-5.2 mmol/L), $\mathrm{HDL}(>1.6 \mathrm{mmol} / \mathrm{L}), \mathrm{TG}(0.5-1.9 \mathrm{mmol} / \mathrm{L}), \operatorname{AspAT}(5-40 \mathrm{U} / \mathrm{L})$, and AlAT (5-40 U/L) were determined by a fully automated Modular P-800 Roche (Diamond Diagnostic, Holliston, USA). LDL was measured indirectly using the Friedewald equation. Insulin (2.0-25.0 mU/L) was determined by a micro-particle enzyme immunoassay (Abbot, Abbot Park, USA). Serum CRP was analyzed by a commercial assay (Dade Behring, Marburg, Germany). The QUICKI was calculated using the inverse of the sum of the logarithms of fasting insulin and fasting glucose levels: $1 /[\log ($ fasting insulin $\mu \mathrm{U} / \mathrm{mL})+\log ($ fasting glucose $\mathrm{mg} / \mathrm{dL})]$. The HOMAIR was calculated according to this formula: fasting insulin $\mu \mathrm{U} / \mathrm{L} \times$ fasting glucose $\mathrm{nmol} / \mathrm{L} / 22.5$.

An important element of the study was the analysis of the serum profiles of 42 AAs in obese patients and their possible changes after weight loss. Fasting plasma was collected for AA analysis in the morning after $10 \mathrm{~h}$ of fasting and was stored in $-80^{\circ} \mathrm{C}$ before analysis. The determination of free AA serum profiles was performed using the fully validated, highly selective liquid chromatography-tandem mass spectrometry method (LC-MS/MS). Samples were prepared using aTRAQ-based methodology (Sciex, Framingham, USA), and then analyzed by a high-performance liquid chromatograph 1260 Infinity (Agilent Technologies, Santa Clara, USA) interfaced to a triple-quadrupole mass spectrometer 4000 QTRAP (Sciex, Framingham, USA). The detailed sample preparation protocol, LC-MS/MS parameters and validation results using serum samples were presented in the previous reports. ${ }^{14,15}$

\section{Anthropometric measures}

For each patient, anthropometric measurements were carried out at baseline and after the 3-week weight reduction program. Height was measured to the nearest centimeter using a stadiometer. Weight and analysis of body composition was performed by Tanita MC 980 MA bodyfat analyzer (TANITA, Tokio, Japan) based on the measurement of bioelectrical impedance, with a tetrapolarfoodpad-style electrode arrangement. The subjects stood on the metal contacts in bare feet, as recommended in the manual. Body mass index was defined as the individual's body mass divided by the square of their height, with the value given in $\mathrm{kg} / \mathrm{m}^{2}$. Waist-hip ratio (WHR) was the ratio of waist circumference to hip circumference, measured in $\mathrm{cm}$ with the use of medical measuring tape (SECA, Hamburg, Germany).

\section{Statistical analysis}

In order to examine changes in the anthropometric and biochemical parameters, as well as free AA serum profiles induced by the weight loss program, the ShapiroWilk test of normality was used in the first step. Variables with normal distribution were then subjected to the paired t-test. For the analysis of variables that were not normally distributed, the Wilcoxon signed-rank test was used. In all tests, a p-value of $\leq 0.05$ was considered to be statistically significant. Additionally, in order to evaluate the influence of a reduction in body weight on the free AA levels in the serum, a principal component analysis (PCA) was conducted. PCA is used to bring out any patterns in the study dataset and to visualize the differences among groups of analyzed samples. Since PCA is conducted without any prior information on sample classification, it is defined as an unsupervised statistical method. Prior to PCA, normalization by sum and by autoscaling of the amino acid concentrations were performed. The statistical analyses were carried out using STATISTICA v. 10.0 (StatSoft, Kraków, Poland) and the MetaboAnalyst 3.0 web portal. ${ }^{16}$

\section{Results}

\section{Anthropometric and biochemical characteristics of the study population}

The differences in the anthropometric and biochemical parameters measured before and after the 3-week weight loss program are presented in Table 1.

After the applied weight loss program, significant decreases in body mass, BMI, waist circumference, and hip circumference were observed. The average reduction in body mass was $6.0 \mathrm{~kg}$ (5.1\%), which significantly decreased the BMI level $\left(39.7 \pm 4.1 \mathrm{~kg} / \mathrm{m}^{2} \mathrm{vs} 37.7 \pm 3.9 \mathrm{~kg} / \mathrm{m}^{2}\right.$; $\mathrm{p}<0.0000)$. The average waist and hip circumferences 
changed from $121.8 \pm 11.7 \mathrm{~cm}$ to $118.0 \pm 11.1 \mathrm{~cm}(\mathrm{p}=0.0000)$ and from $129.0 \pm 9.1 \mathrm{~cm}$ to $126.0 \pm 8.3 \mathrm{~cm}(\mathrm{p}=0.0000)$, respectively. After the 3-week program, we observed an 8.7\% reduction in fat mass. A reduction in muscle mass and total body water was also noticed in the study group (Table 1).

Table 1. Anthropometric characteristics, biochemical parameters and free AA concentrations of the study population before and after the weight loss program

\begin{tabular}{|c|c|c|c|c|c|}
\hline \multirow{2}{*}{ Parameter } & \multirow{2}{*}{$\frac{\text { Before the program }}{\text { mean } \pm S D}$} & \multirow{2}{*}{$\frac{\text { After the program }}{\text { mean } \pm \mathrm{SD}}$} & \multicolumn{2}{|c|}{ After - before the program } & \multirow{2}{*}{ p-value* } \\
\hline & & & mean $\pm \mathrm{SD}$ & $\%$ difference & \\
\hline \multicolumn{6}{|c|}{ Anthropometric parameters } \\
\hline Body weight $[\mathrm{kg}]$ & $116.1 \pm 14.8$ & $110.1 \pm 13.6$ & $-6.0 \pm 3.0$ & -5.1 & $<0.0000^{* *}$ \\
\hline BMI [kg/m²] & $39.7 \pm 4.1$ & $37.7 \pm 3.8$ & $-2.0 \pm 1.0$ & -5.2 & $<0.0000^{* *}$ \\
\hline Waist circumference $[\mathrm{cm}]$ & $121.8 \pm 11.7$ & $118.0 \pm 11.1$ & $-3.8 \pm 2.8$ & -3.2 & $0.0000^{* *}$ \\
\hline Hip circumference $[\mathrm{cm}]$ & $129.0 \pm 9.1$ & $126.0 \pm 8.3$ & $-3.0 \pm 1.6$ & -2.3 & $0.0000^{* *}$ \\
\hline Fat mass [kg] & $46.3 \pm 8.7$ & $42.2 \pm 8.3$ & $-4.1 \pm 2.0$ & -8.7 & $0.0000^{* *}$ \\
\hline $\mathrm{FFM}[\mathrm{kg}]$ & $69.8 \pm 11.5$ & $67.9 \pm 11.6$ & $-1.9 \pm 2.1$ & -2.8 & $0.0002^{* *}$ \\
\hline Muscle mass [kg] & $66.3 \pm 10.9$ & $64.4 \pm 11.2$ & $-1.9 \pm 2.0$ & -2.9 & $0.0001^{* *}$ \\
\hline TBW $[k g]$ & $50.0 \pm 8.7$ & $48.7 \pm 8.8$ & $-1.3 \pm 2.2$ & -2.7 & $0.0064^{* *}$ \\
\hline \multicolumn{6}{|c|}{ Biochemical parameters } \\
\hline Triglycerides [mg/dL] & $152.7 \pm 87.3$ & $133.6 \pm 58.1$ & $-19.1 \pm 48.9$ & -12.5 & 0.1004 \\
\hline $\mathrm{LDL}[\mathrm{mg} / \mathrm{dL}]$ & $119.2 \pm 35.6$ & $105.9 \pm 32.9$ & $-13.3 \pm 32.9$ & -11.3 & 0.0560 \\
\hline CRP $[\mathrm{mg} / \mathrm{L}]$ & $4.1 \pm 2.7$ & $4.0 \pm 3.2$ & $-0.1 \pm 3.0$ & -3.4 & 0.3606 \\
\hline Glucose $[\mathrm{mg} / \mathrm{dL}]$ & $108.9 \pm 19.6$ & $101.9 \pm 12.2$ & $-7.0 \pm 14.3$ & -6.4 & $0.0082^{* *}$ \\
\hline Insulin $[\mu \mathrm{U} / \mathrm{mL}]$ & $21.3 \pm 16.4$ & $18.0 \pm 15.8$ & $-3.3 \pm 4.7$ & -15.7 & $0.0006^{* *}$ \\
\hline HOMA-IR & $6.1 \pm 5.4$ & $4.8 \pm 4.8$ & $-1.3 \pm 1.8$ & -20.4 & $0.0029^{* *}$ \\
\hline QUICKI & $0.2 \pm 0.0$ & $0.2 \pm 0.0$ & $0.0 \pm 0.0$ & 5.6 & $0.0244^{* *}$ \\
\hline ALAT [U/L] & $35.3 \pm 17.9$ & $31.6 \pm 18.0$ & $-3.7 \pm 9.4$ & -10.4 & $0.0355^{* *}$ \\
\hline AspAT [U/L] & $25.3 \pm 13.2$ & $24.7 \pm 16.3$ & $-0.6 \pm 6.2$ & -2.5 & 0.2373 \\
\hline \multicolumn{6}{|c|}{ Amino acids $[\mu \mathrm{M}]$} \\
\hline 1-methylhistidine & $6.09 \pm 4.77$ & $8.03 \pm 6.30$ & $1.96 \pm 7.60$ & 32.0 & 0.1451 \\
\hline 3-methylhistidine & $3.20 \pm 1.12$ & $3.61 \pm 1.59$ & $0.42 \pm 1.49$ & 13.0 & 0.1322 \\
\hline a-aminoadipic acid & $0.79 \pm 0.40$ & $0.79 \pm 0.41$ & $-0.0001 \pm 0.43$ & 0.0 & 0.7701 \\
\hline a-amino-n-butyric acid & $25.05 \pm 6.06$ & $30.00 \pm 6.20$ & $4.95 \pm 6.21$ & 19.8 & $0.0007^{* *}$ \\
\hline Alanine & $382.44 \pm 112.01$ & $452.68 \pm 128.73$ & $70.24 \pm 150.26$ & 18.4 & 0.0315 \\
\hline Arginine & $65.72 \pm 17.40$ & $71.04 \pm 12.21$ & $5.32 \pm 15.47$ & 8.1 & 0.1055 \\
\hline Asparagines & $38.37 \pm 8.15$ & $40.07 \pm 5.78$ & $1.70 \pm 8.38$ & 4.4 & 0.3306 \\
\hline Aspartic acid & $11.63 \pm 3.73$ & $9.83 \pm 2.38$ & $-1.80 \pm 3.42$ & -15.5 & $0.0169^{* *}$ \\
\hline$\beta$-aminoisobutyric acid & $2.64 \pm 0.88$ & $2.88 \pm 1.01$ & $0.24 \pm 0.77$ & 8.9 & 0.1462 \\
\hline$\beta$-alanine & $22.42 \pm 4.29$ & $22.01 \pm 2.94$ & $-0.41 \pm 3.39$ & -1.8 & 0.5573 \\
\hline Citrulline & $18.56 \pm 7.53$ & $22.26 \pm 8.30$ & $3.70 \pm 7.56$ & 19.9 & $0.0251^{* *}$ \\
\hline Cystine & $47.11 \pm 14.87$ & $49.23 \pm 13.17$ & $2.13 \pm 13.18$ & 4.5 & 0.4373 \\
\hline Ethanolamine & $7.11 \pm 1.87$ & $6.96 \pm 1.37$ & $-0.15 \pm 1.38$ & -2.1 & 0.5994 \\
\hline Glutamine & $444.59 \pm 85.25$ & $503.76 \pm 75.65$ & $59.17 \pm 79.85$ & 13.3 & $0.0014^{* *}$ \\
\hline Glutamic acid & $48.13 \pm 14.44$ & $45.03 \pm 13.90$ & $-3.10 \pm 13.86$ & -6.5 & 0.2842 \\
\hline Glycine & $221.77 \pm 53.58$ & $261.28 \pm 54.53$ & $39.51 \pm 43.12$ & 17.8 & $0.0002^{* *}$ \\
\hline Histidine & $63.32 \pm 13.77$ & $63.06 \pm 7.93$ & $-0.26 \pm 11.04$ & -0.4 & 0.9078 \\
\hline Hydroxylysine & $8.18 \pm 3.51$ & $9.89 \pm 3.63$ & $1.71 \pm 5.12$ & 20.9 & $0.0199^{* *}$ \\
\hline Isoleucine & $65.08 \pm 13.85$ & $72.20 \pm 18.54$ & $7.13 \pm 16.62$ & 11.0 & $0.0469^{* *}$ \\
\hline Leucine & $117.50 \pm 25.05$ & $120.41 \pm 27.43$ & $2.91 \pm 29.53$ & 2.5 & 0.8639 \\
\hline Lysine & $181.17 \pm 40.80$ & $183.58 \pm 34.84$ & $2.41 \pm 42.42$ & 1.3 & 0.7833 \\
\hline Methionine & $20.10 \pm 5.31$ & $20.45 \pm 4.54$ & $0.35 \pm 5.55$ & 1.8 & 0.7584 \\
\hline
\end{tabular}


Table 1 (cont.).

\begin{tabular}{|l|c|c|c|c|c|}
\multirow{2}{*}{\multicolumn{1}{c|}{ Parameter }} & Before the program & After the program & \multicolumn{2}{|c|}{ After - before the program } & p-value* \\
\cline { 2 - 6 } & mean \pm SD & mean \pm SD & mean \pm SD & \% difference & 0.2010 \\
\hline Ornithine & $65.91 \pm 16.94$ & $71.03 \pm 17.76$ & $5.12 \pm 19.05$ & 7.8 & 0.7394 \\
\hline Phosphoethanolamine & $1.79 \pm 0.62$ & $1.74 \pm 0.46$ & $-0.04 \pm 0.64$ & -2.5 & 0.3642 \\
\hline Phenylalanine & $50.60 \pm 9.04$ & $48.66 \pm 7.59$ & $-1.94 \pm 10.24$ & -3.8 & $0.0014^{* *}$ \\
\hline Proline & $146.42 \pm 44.28$ & $190.51 \pm 53.79$ & $44.09 \pm 66.76$ & 30.1 & $0.0040^{* *}$ \\
\hline Sarcosine & $1.31 \pm 0.66$ & $1.62 \pm 0.86$ & $0.31 \pm 0.48$ & 23.9 & 0.2873 \\
\hline Serine & $106.88 \pm 24.21$ & $112.35 \pm 25.04$ & $5.47 \pm 24.60$ & 5.1 & 0.3053 \\
\hline Taurine & $93.74 \pm 25.84$ & $88.95 \pm 21.94$ & $-4.79 \pm 22.37$ & -5.1 & $0.0148^{* *}$ \\
\hline Threonine & $91.94 \pm 21.87$ & $109.08 \pm 31.94$ & $7.14 \pm 31.85$ & 18.6 & 0.8854 \\
\hline Tryptophan & $42.41 \pm 10.99$ & $42.16 \pm 7.24$ & $-0.25 \pm 8.35$ & -0.6 & 0.081 \\
\hline Tyrosine & $42.51 \pm 11.20$ & $46.81 \pm 14.16$ & $4.30 \pm 11.54$ & 10.1 & 0.0892 \\
\hline Valine & $211.71 \pm 42.25$ & $227.04 \pm 37.33$ & $15.33 \pm 42.31$ & 7.2 & 0 \\
\hline
\end{tabular}

* obtained based on paired t-test or Wilcoxon test; ** statistically significant; BMI - body mass index; FFM - free fat mass, TBW - total body water; LDL - low-density lipoprotein; CRP - C-reactive protein; HOMA-IR - homeostatic model assessment of insulin resistance; QUICKI - quantitative insulin sensitivity check index; AIAT - alanine aminotransferase; AspAT - aspartate aminotransferase.

glucose

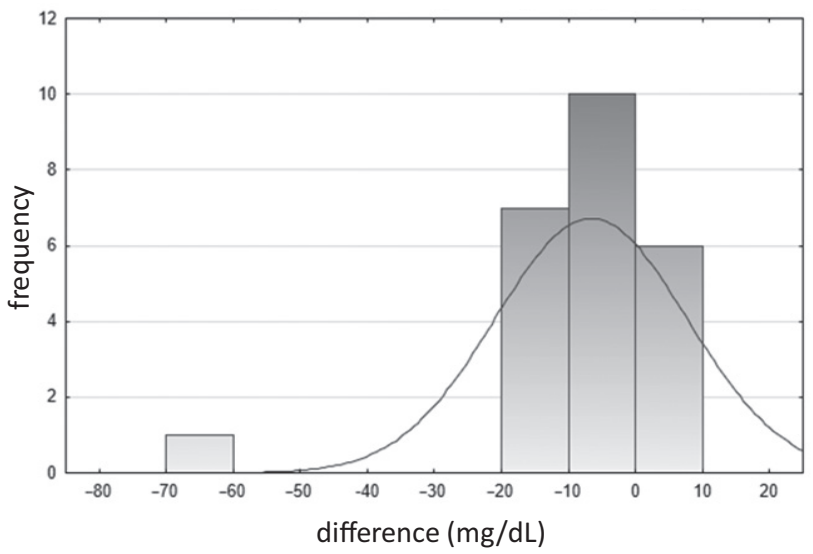

HOMA-IR

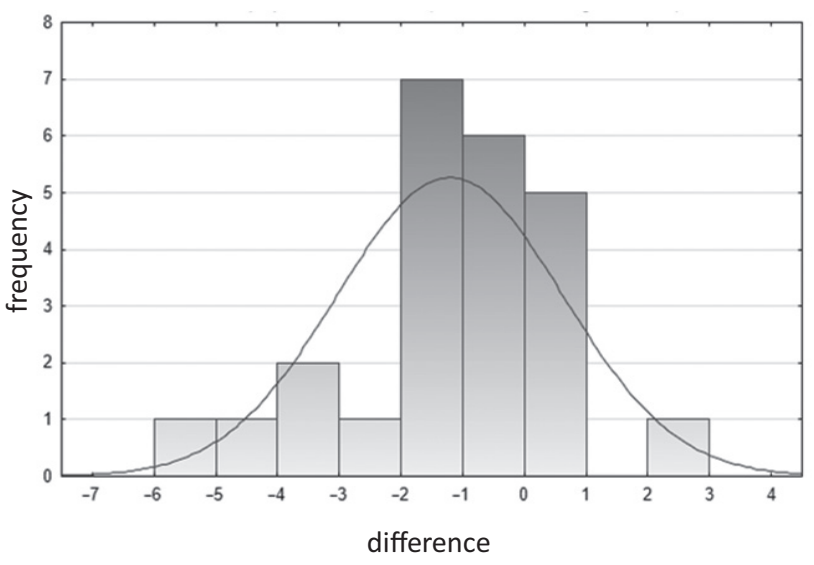

insulin

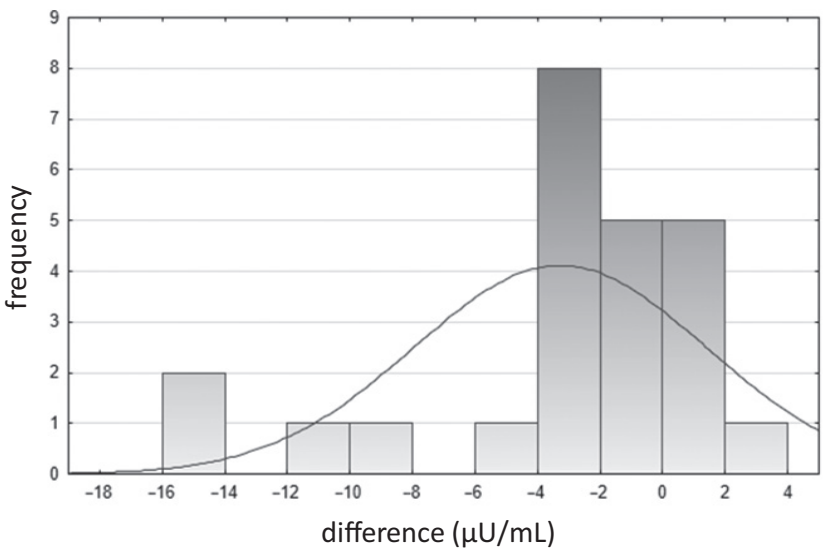

QUICKI

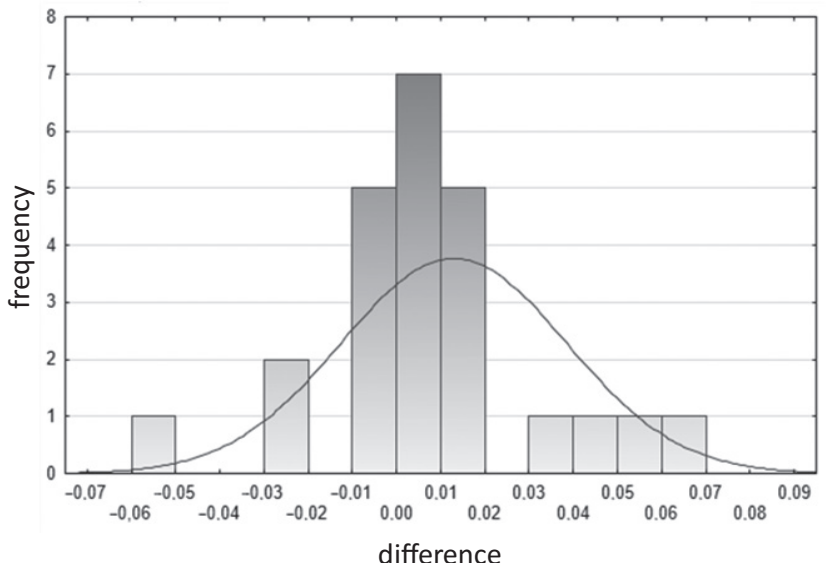

Fig. 1. Histograms of the differences in parameters associated with carbohydrate metabolism measured before and after the 3-week weight loss program HOMA-IR - homeostatic model assessment of insulin resistance; QUICKI - quantitative insulin sensitivity check index.

Baseline median fasting blood glucose levels in patients with obesity were elevated compared to the values after body mass reduction (Table 1). Similarly, the fasting insulin levels in patients at baseline were significantly higher than at the end of the study. We observed positive changes in HOMA-IR after weight loss (Fig. 1). Other important changes observed after weight loss were the reductions in TG concentration (12.5\%), the LDL-cholesterol level (11.4\%) and CRP (3.4\%); however, those differences were not statistically significant. 


\section{AA profiles of serum}

The analysis of free AA profiles in the serum samples collected before and after the 3-week weight loss program revealed a number of significant changes in the metabolic profile of the patients. Although the applied methodology allows for measurements of 42 free AAs, both proteinogenic and non-proteinogenic, not all of the metabolites occurred in measurable concentrations in the samples. For this reason, the following 9 AAs were excluded from further statistical analysis: phosphoserine, argininosuccinic acid, homocitrulline, anserine, carnosine, homocysteine, $\gamma$-amino-n-butyric acid, cystathionine, and $\delta$-hydroxylysine. The remaining 33 metabolites, along with their concentrations as measured in the analyzed serum samples, are listed in Table 1 . The levels of 10 AAs ( $\alpha$-amino-n-butyric acid, alanine, citrulline, glutamine, glycine, hydroxyproline, isoleucine, proline, sarcosine, and threonine) were significantly higher after weight loss compared with their values before the program. Only aspartic acid was lower in patients after the program. According to the univariate statistical analyses performed, the highest differences in serum levels were observed for the following metabolites: glycine $(\mathrm{p}=0.0002), \alpha$-amino-n-butyric acid $(\mathrm{p}=0.0007)$, proline $(\mathrm{p}=0.0014)$, and glutamine $(\mathrm{p}=0.0014)$.

The influence of weight loss on AA profiles was also investigated using multivariate statistical analysis - PCA - which involves a group of variables simultaneously. Free AA profiles were analyzed by PCA to determine any clusters with respect to the 2 groups of samples: before and after intervention. In Fig. 2, points corresponding to the analyzed serum samples are contained in the space spanned by the first 2 principal components (PC 1 vs PC 2). Although the 2 groups of samples did not form separate clusters, a partial separation between samples was observed (Fig. 2). The results of PCA indicate that the changes in free AA profiles were strong enough to cause grouping of the samples according to their inclusion in one of the groups studied.

\section{Discussion}

This paper presents a comprehensive picture of the metabolic changes in Polish patients with obesity after a 3-week weight loss program. Although the duration of the body mass reduction program was relatively short, it is noteworthy that the weight reduction was carried out under strictly controlled conditions. All patients were treated with the same type of diet prepared by dietetic food caterers and the level of compliance remained under supervision during the entire 3 weeks. The implemented program of physical exercise was also the same in all study participants.

The weight loss achieved in all patients during the 3-week program (based on a hypocaloric diet of a strictly defined macronutrient composition and physical activity) was very satisfactory and it affected the measured

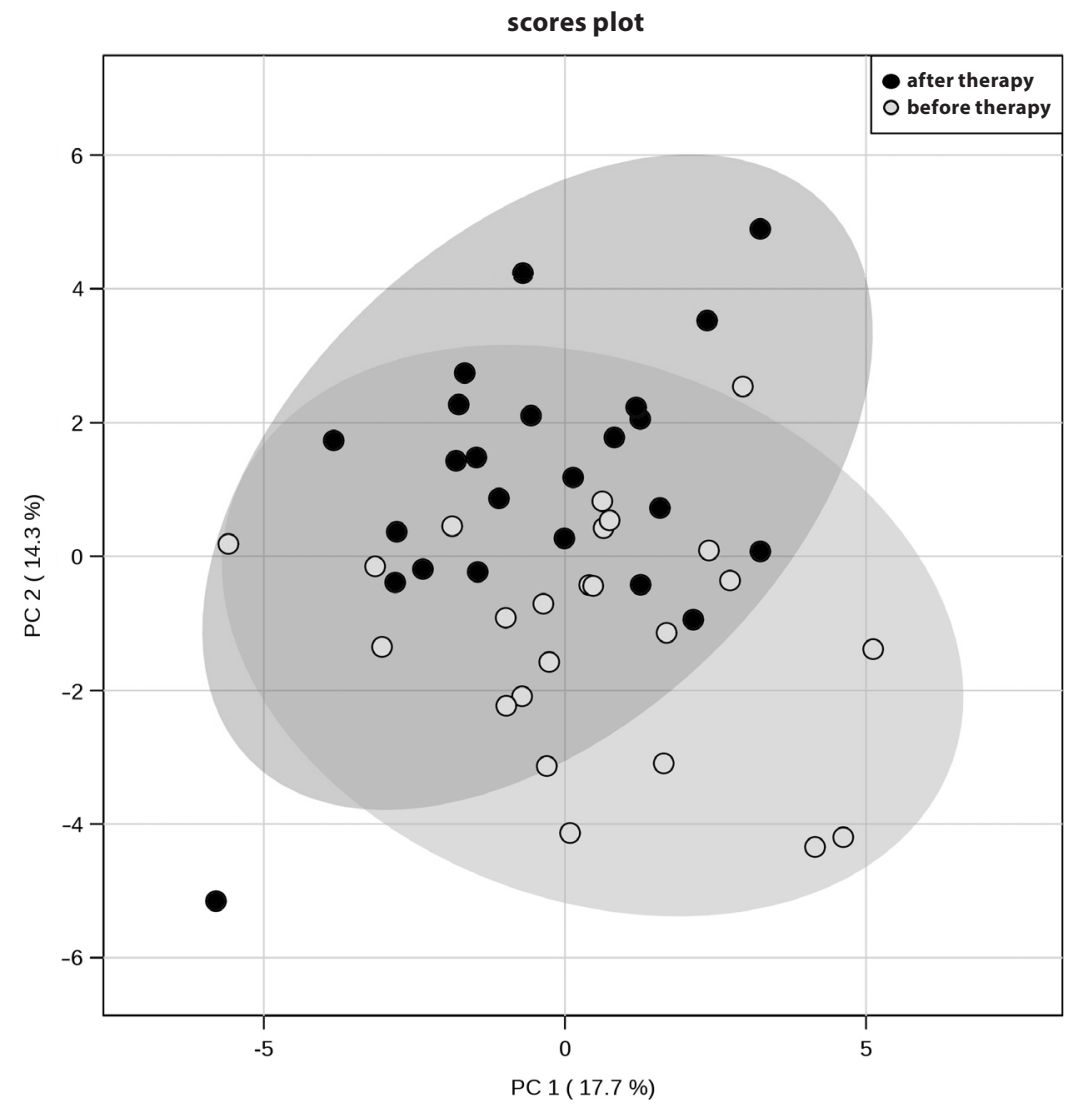

Fig. 2. Score plot from PCA model classifying the serum samples according to their inclusion in one of the studied groups (before or after weight loss)

PCA - principal component analysis. 
metabolic parameters and body mass composition. It has been already demonstrated that a $5 \%$ weight loss reduces or eliminates disorders associated with obesity; it improves both TC and TG, and causes a reduction in plasma glucose level. ${ }^{17}$ Similar trends were observed in this study, even though the body mass reduction was achieved after a short-term program. The TG and LDL-cholesterol levels were reduced in response to weight loss, though those differences were not statistically significant. As a result of weight reduction, positive changes in carbohydrate profiles were observed (Fig. 1). We noticed positive changes in HOMA-IR and QUICKI after weight loss. The HOMAIR and QUICKI are widely validated and applied in the estimation of insulin sensitivity. ${ }^{18}$ High HOMA-IR and low QUICKI were independently and significantly associated with an increased risk of impaired glucose tolerance (IGT) and type 2 diabetes. The HOMA-IR cut off value which correlates with an increased risk of metabolic and cardiovascular diseases was estimated in several studies, in which its dependence on age, gender and ethnicity was noted. ${ }^{19}$ In the study population, HOMA-IR was elevated, but we observed a reduction after the intervention ended $(\mathrm{p}=0.0029)$ (Table 1$)$.

Our study involved the application of a targeted metabolomic approach focused on the profiles of free AAs and their derivatives in the serum of adult Polish patients with obesity at baseline and after 3 weeks of a weight loss program. The previous studies described the changes in biochemical parameters after weight loss, but only a few studies assessed the impact of the reduction in body mass and body composition changes on free AA profiles. The available conclusions on this relationship mainly come from studies of patients after bariatric surgery or long-term weight reduction programs. However, it has been shown that bariatric surgery caused differential metabolic changes in AA profiles of patients with obesity compared with dietary intervention, despite identical weight loss of the subjects. ${ }^{20,21}$ Additionally, only a few studies investigated the effect of weight reduction on the AA levels in time points shorter than 1 year. An intriguing issue is the dynamics of changes in the metabolome of people with obesity during weight loss. Moreover, the previous studies on changes in AA profiles during weight loss were conducted on a group of patients of varying races (especially among Asians and Americans), which may have influenced the observations. The results obtained in the previous studies may have also depended on the technique of metabolomic assays. The advantages of the analytical methodology used in the current study were confirmed in the available literature and include high sensitivity and specificity, short time of analysis and a very wide range of analytes, since it enables the quantification of 42 AAs, both proteinogenic and non-proteionogenic. ${ }^{15,16}$ As 9 AAs of the identified 42 AAs occurred in concentrations below measurable values, the final analysis included 33 metabolites.
In this study, we observed that the levels of $10 \mathrm{AAs}$ ( $\alpha$-amino-n-butyric acid, alanine, citrulline, glutamine, glycine, hydroxyproline, isoleucine, proline, sarcosine, and threonine) had increased significantly after weight loss compared to their values before the program, while the aspartic acid level had decreased. According to the univariate statistical analyses performed, the highest differences in serum levels were observed for the following metabolites: glycine, $\alpha$-amino-n-butyric acid, proline, and glutamine (Table 1). An elevation in the glycine and threonine levels was also observed in a population of overweight, older German adults, following an 8-week calorie-restricted (15\% less than daily energy requirements) weight loss and physical activity program. ${ }^{22}$

An elevation of the BCAA levels has been reported in humans with obesity and in animal models of obesity, and many studies have described a decrease in the levels of BCAAs after weight loss. ${ }^{12,22-24}$ Some studies demonstrated a decrease in concentrations of BCAA and metabolites derived from BCAA oxidation after bariatric surgery. ${ }^{25,26}$ The increased plasma BCAA concentration may be the result of the impaired BCAA-catabolizing capacity of the adipose tissue, an improper high-fat and highprotein diet (dietary protein comprised of $>20 \%$ BCAA) and increased catabolic pathways of BCAAs. ${ }^{13,20,27,28}$ So far, only a few studies rated the changes in the BCAA levels after weight loss through diet and physical activity, but those studies were conducted on different populations (especially Caucasian and Japanese subjects) and used another model of body mass reduction program (based on nutritional recommendation and lifestyle intervention, but not a strictly composed diet). A 7-year prospective KORA study on a group of Caucasian patients with obesity showed a significant correlation between the average percentage increase in body weight and the increase in the BCAA, phenylalanine, tyrosine, and glutamate levels. ${ }^{29}$ In our study, we observed that the valine, isoleucine and leucine levels increased (Table 1) despite the body mass reduction. Tochikubo et al. noted that a 3 -month diet and exercise intervention based on individual dietary guidance caused a normalization in circulating BCAA. ${ }^{30}$ In their study on Japanese subjects, Tochikubo et al. first observed that the initial plasma concentrations of AAs predict the success or lack thereof of a 3-month standardized diet and exercise weight loss program. ${ }^{30}$

The 1-year body mass reduction program based on lifestyle intervention (changes in diet and exercise habits) conducted by Reinehr et al., showed an increased level of serine, glutamine and methionine, but not proline, after the body mass reduction in a group of German children with obesity. ${ }^{31}$ Wahl et al. described that the proline levels were reduced in children suffering from obesity compared to children with normal weight. ${ }^{26}$ Moreover, Wahl et al. demonstrated that methionine and glutamine were diminished at baseline in children with obesity who reduced weight during lifestyle intervention, as compared with 
children without the body mass reduction in the same kind of intervention. ${ }^{26}$ The same relationship was described by Pathmasiri et al. in a study on an adult population with obesity, which leads to the hypothesis that dysregulation in the AA levels might be a consequence of poor lifestyle habits (diet and exercise). ${ }^{32}$

Some inconsistencies between our findings and the data obtained from previous studies were found. These differences may have been caused by different duration and methods of the weight reduction program, or by additional factors, such as the ethnicity or age of the study population, baseline BMI values and the level of weight loss achieved, or the protein content of the patients' normal diet. The current study was conducted on Polish patients (Caucasian race), while the previous study involved mainly Asian populations. In our study, the subjects were hospitalized for 3 weeks in a controlled environment. Additionally, each patient received the same type of a hypocaloric diet based on a $25-30 \%$ reduction in caloric dietary intake compared to total energy requirement. To eliminate the possible effect of diet composition on the levels of circulating AAs, a diet with an identical composition and sources of macronutrients (especially proteins) was administered to all patients. Moreover, the mean BMI in our study population after the weight loss program was lower, but still above $35 \mathrm{~kg} / \mathrm{m}^{2}$, which could account for the differences in the AA changes in our study compared to previous data.

\section{Conclusions}

This study is the first to describe the changes in 42 free AA profiles during a 3-week body mass reduction program in adult Polish patients with obesity. Our study shows that the metabolic profile of patients undergoing a strictly controlled weight reduction program were characterized by high dynamics of change and were visible after 3 weeks of body mass reduction.

The 3-week diet and physical activity program caused significant changes in body mass and biochemical parameters (e.g., the fasting glucose and insulin levels, HOMA-IR, CRP, and TG) and changes in free AA profiles. We observed that the levels of 10 out of the 42 AAs measured were significantly higher after weight loss. The highest differences in serum levels were observed for the following metabolites: glycine, $\alpha$-amino-n-butyric acid, proline, and glutamine. Some variations between our findings and the data obtained from previous studies were found. These differences may have been caused by different duration and methods of the weight reduction program, or by additional factors, such as the ethnicity or age of the study population, baseline BMI values and the level of weight loss achieved, the protein content of the patients' normal diet, or the impact of physical activity on the metabolism of AA. To better understand these changes, future studies should involve a long-term program with more patients.

\section{References}

1. Baboota R, Bishnoi M, Ambalam P, et al. Functional food ingredients for the management of obesity and associated co-morbidities: A review. J Funct Foods. 2013;5:997-1012.

2. Conroy KP, Davidson IM, Warnock M. Pathogenic obesity and nutraceuticals. Proc Nutr Soc. 2011;70:426-438.

3. Patti GJ, Yanes O, Siuzdak G. Innovation: Metabolomics: The apogee of the omicstrilogy. Nat Rev Mol Cell Biol. 2012;13:263-269.

4. Wu G. Amino acids: Metabolism, functions and nutrition. Amino Acids 2009;37:1-17.

5. Wang TJ, Larson MG, Vasan RS. Metabolite profiles and the risk of developing diabetes. Nat Med. 2011;17:448-453.

6. Morris C, O'Grada C, Ryan M, et al. The relationship between BMI and metabolomic profiles: A focus on amino acids. Proc Nutr Soc. 2012;71:634-638.

7. Wery JP. Application of proteomics technologies to biomarker discovery and development: Challenges and solutions. Curr Sep. 2007;22:15-17.

8. Mayeux R. Biomarkers: Potential uses and limitations. NeuroRx. 2004; 1:182-188.

9. Chevalier S, Marliss EB, Morais JA, et al. Whole-body protein anabolic response is resistant to the action of insulin in obese women. $A m$ J Clin Nutr. 2005;82:355-365.

10. Newgard CB, An J, Bain JR, et al. A branched-chain amino acid-related metabolic signature that differentiates obese and lean humans and contributes to insulin resistance. Cell Metab. 2009;9:311-326.

11. McCormack SE, Shaham O, McCarthy MA, et al. Circulating branchedchain amino acid concentrations are associated with obesity and future insulin resistance in children and adolescents. Pediatr Obes. 2013;8:52-61.

12. Yamakado M, Tanaka T, Nagao K, et al. Plasma amino acid profile is associated with visceral fat accumulation in obese Japanese subjects. Clin Obes. 2012;2:29-40.

13. Marti FPJ, Montoliu I, Collino S, et al. Topographical body fat distribution links to amino acid and lipid metabolism in healthy non obese women. PLoS One. 2013;8:e73445.

14. Held PK, White L, Pasquali M. Quantitative urine amino acid analysis using liquid chromatography tandem mass spectrometry and aTRAQ reagents. J Chromatogr B. 2011;879:2695-2703.

15. Matysiak J, Dereziński P, Klupczyńska A, et al. Effects of a honeybee sting on the serum free amino acid profile in humans. PLoS One. 2014;9:e103533.

16. Xia J, Sinelnikov IV, Han B, et al. MetaboAnalyst 3.0: Making metabolomics more meaningful. Nucleic Acids Res. 2015;43:W251-257.

17. Blackburn G. Effect of degree of weight loss on health benefits. Obes Res. 1995;3:211-216.

18. Foss-Freitas MC, Foss MC. Comparison of HOMA, QUICKI and forearm metabolic data in humans. Braz J Med Biol Res. 2004;37:663-668.

19. Song Y, Manson J, Tinker L. Insulin sensitivity and insulin secretion determined by homeostasis model assessment and risk of diabetes in a multiethnic cohort of women. Diabetes Care. 2007;30:1747-1752.

20. Oberbach A, von Bergen M, Bluher S. Combined serum proteomic and metabolomic profiling after laparoscopic sleeve gastrectomy in children and adolescents. J Laparoendosc Adv Surg Tech A. 2012;22: 184-188.

21. Laferrere B, Reilly D, Arias S, et al. Differential metabolic impact of gastric bypass surgery versus dietary intervention in obese diabetic subjects despite identical weight loss. Sci Transl Med. 2011;3:80-82.

22. Perez-Cornago A, Brennan L, Ibero-Baraibar I, et al. Metabolomics identifies changes in fatty acid and amino acid profiles in serum of overweight older adults following a weight loss intervention. JPhysiol Biochem. 2014;70:593-602.

23. Shah SH, Crosslin DR, Haynes CS. Branched-chain amino acid levels are associated with improvement in insulin resistance with weight loss. Diabetologia. 2012;55:321-330.

24. Herman MA, She P, Peron OD. Adipose tissue branched chain amino acid (BCAA) metabolism modulates circulating BCAA levels. J Biol Chem. 2010;285:11348-11356.

25. Lips MA, van Klinken JB, van Harmelen V. Roux-en-Y gastric bypass surgery, but not calorie restriction, reduces plasma branched-chain amino acids in obese women independent of weight loss or the presence of type 2 diabetes. Diabetes Care. 2014;37:3150-3156. 
26. Wahl S, Yu Z, Kleber M, et al. Childhood obesity is associated with changes in the serum metabolite profile. Obes Facts. 2012;5:660-670.

27. O'Connell TM. The complex role of branched chain amino acids in diabetes and cancer. Metabolites. 2013;3:931-945.

28. She P, Van Horn C, Reid,T et al. Obesity-related elevations in plasma leucine are associated with alterations in enzymes involved in branched-chain amino acid metabolism. Am J Physiol Endocrinol Metab. 2007;293:E1552-1563.

29. Wahl S, Vogt S, Stückler F, et al. Multi-omic signature of body weight change: Results from a population-based cohort study. BMC Medicine. 2015;13:48.
30. Tochikubo $\mathrm{O}$, Nakamura $\mathrm{H}$, Jinzu $\mathrm{H}$, et al. Weight loss is associated with plasma free amino acid alterations in subjects with metabolic syndrome. Nutrition \& Diabetes. 2016;6:e197;1-6.

31. Reinehr T, Wolters B, Knop C, et al. Changes in the serum metabolite profile in obese children with weight loss. Eur J Nutr. 2015,54:173-181.

32. Pathmasiri W, Pratt KCD, Lutes L, et al. Integrating metabolomic signatures and psychosocial parameters in responsivity to an immersion treatment model for adolescent obesity. Metabolomics. 2012;8: 1037-1051. 